\title{
Imaging of Meningioma
}

\author{
Duma Ratna Sari Nasution ${ }^{1,2}$, Ahmad Brata Rosa ${ }^{1}$, Robert Sinurat ${ }^{1}$, Sanggam \\ Hutagalung ${ }^{1,3}$ \\ ${ }^{1}$ Deli Serdang General Hospital Lubuk Pakam, North Sumatera, Indonesia \\ ${ }^{2}$ Education Comittee of FK UMSU Hospital Teaching \\ ${ }^{3}$ Lecturer of Medical Faculty Indonesian Methodist University
}

Correspondence email: sanggam1973@gmail.com

\begin{abstract}
Meningioma is one of the tumor in the brains that is extra-axial, which means it is not originated from the brain cell. We report a case of a 60 -year-old female, that was admitted to the emergency room (ER) of a secondary referral hospital, due to sudden weakness of upper and lower right extremities since 2 days prior. She had been experiencing a feeling of fullness in the left frontal head since the previous 3 months, along with some headache. She admitted to have had diabetes for the last 2 years. She also reported family history of brain tumor. Neurosurgeon has diagnosed the tumor as a meningioma with differential diagnose a metastatic tumor. A non contrast CT scan has found a meningioma. After being diagnosed, the patient underwent a craniotomy to remove the tumor. The histopathology finding confirmed the diagnosis of meningioma (WHO Grade I). Meningiomas are mostly hyperattenuating to surrounding brain parenchyma, while roughly one fourth are isodense. Overt calcification as in this case is usually seen in psammomatous and meningothelial. Well collaboration between clinician, radiologist, and histopathologist is the key to ensure patient's recovery and safety.
\end{abstract}

Keywords: meningioma, head CT scan, calcification

\section{BACKGROUND}

Meningioma is one of the brain tumors that comes from the extra-axial, which means it is not of the brain cell origin. People who are suffered from this tumor usually develop headache, fullness in the head, nausea, vomiting, and even hemiplegia. It is very important to determine whether a hemiparesis is due to a cerebrovascular accident or a space occupying lesion effect (i.e. tumor). Head CT scan is the key to solve the problem in early case. In many literatures, meningioma can be seen through head scan as a solid hyperdense image, either solitaire or multiple. Overt calsifications can be found in several cases, that can assist us in deciding whether it is a benign or a malignant one. Neurosurgeons must be aware of both the typical and atypical imaging appearances of meningiomas, as there is some correlation with different histologic types of tumor.

Significant factors contributing to the recurrence include histologic types (atypical or malignant) and tumor contrast enhancement on CT scans. Physicians must gather the information from clinical appearance, radiology, and histopathology findings to confirm the diagnosis. 


\section{CASE REPORT}

A 60-year-old female was brought to the ER of a secondary referral hospital in Deli Serdang with sudden weakness of upper and lower right extremities. This has occurred since the last 2 days without losing her consciousness. She has been having fullness in the left frontal head since the previous 3 months, sometimes accompanied by headache. She has also been diagnosed with a diabetes for the last 2 years. Her blood pressure has increased since the occurrence of the hemiplegic. She had a sister that also suffered from brain tumor, and has passed away a year ago. Due to the hemiparesis, the general practitioner sent her to a neurologist, who then performed a CT scan, which eventually revealed a meningioma. The neurologist then sent this patient to a neurosurgeonfor further management. The neurosurgeon diagnosed the tumor as a meningioma with differential diagnosis of a metastatic tumor.
In the non contrast $\mathrm{CT}$ scan with $5 \mathrm{~mm}$ axial cut per slice (Figure 1), a hyperdense mass with the size of $32,1 \mathrm{~mm} \times 30,6 \mathrm{~mm}$ is seen on the left frontal of the brain, whereas the rest is normal. Conclusion: a space occupying lesion with cerebral oedema, size $32,1 \mathrm{~mm} \times 30,6 \mathrm{~mm}$ on the left frontal, with differential diagnosis of meningioma.

Afterward, the patient underwent a craniotomy to remove the tumor. After the tumor removal, the tumor was sent to Pathology Laboratory to examined histopathologically. Macroscopic examination showed a spongy mass sized $2,5 \mathrm{~cm} \times 2,5 \mathrm{~cm} \times 2 \mathrm{~cm}$, grey, and wellcircumscribed. Microscopic examination revealed groups of diffused, wide Psammoma bodies (PB) and spindle shaped epithelial cells with fine chromatin, and regular membrane. There is no malignant sign found in the sample. The conclusion was meningioma (WHO Grade I). 
E-ISSN: 2528-410X

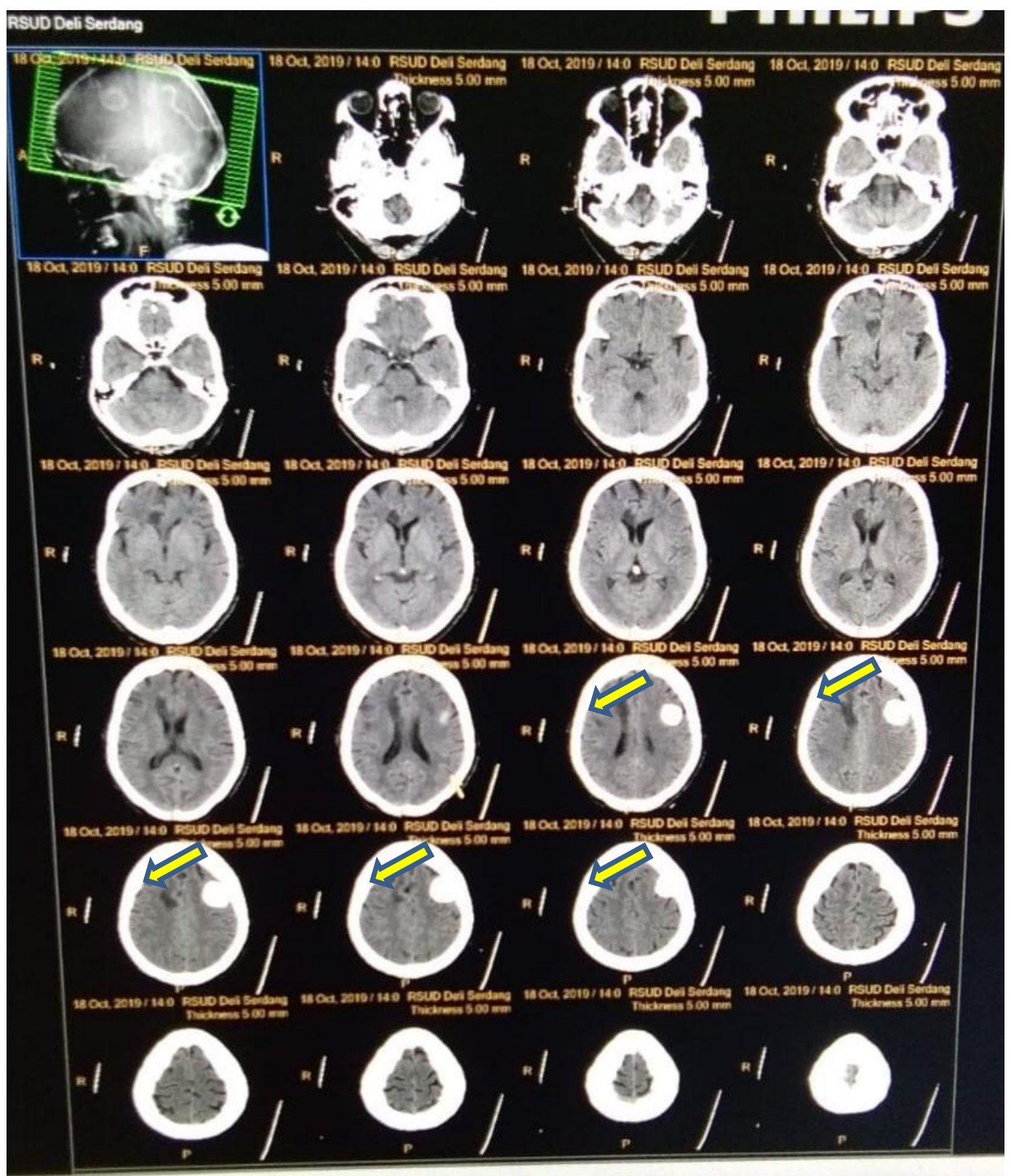

Figure 1. Head CT scan. Arrows show hyperdense mass, well marginated, and calcification 


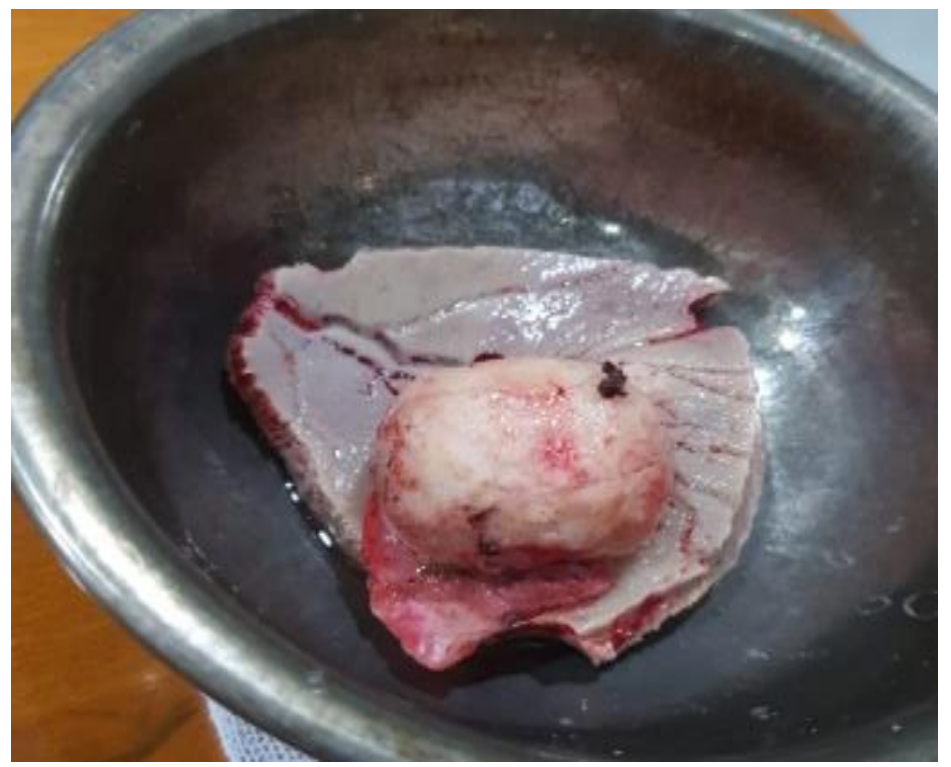

Figure 2. Tumor mass

\section{DISCUSSION}

It is clear that meningioma imaging typically appears as a hyperdense feature with duratail. Computed tomography (CT) scanning is frequently utilized in the assessment of meningiomas. Typical features on unenhanced images include a well-circumscribed, smoothly marginated extra-axial mass abutting the dura. The mass can also be enhanced using a contrast CT as an additional work-up. Meningingioma may be similar to other types of metastatic cancer, therefore, it is important to determine the origin of the cancer. Metastatic tumor can be single or multiple well-circumscribed mass. ${ }^{1,2}$

Approximately $90 \%$ of meningiomas are demonstrated on CT scans. The main role of CT scanning, as opposed to MRI, is to demonstre the adjacent bone changes and calcification within the lesion. Approximately two third of meningiomas are invasive to surrounding brain parenchyma, while the remaining small percentage is isodense. A rare groups of meningiomas (the lipoblastic subtype) contain fat and are thus hypoattenuating. ${ }^{1-3}$

Calcification is another common finding, seen in approximately $20-25 \%$ of cases. The CT nature of the calcification may be nodular, fine and punctate, or dense. Surrounding parenchymal vasogenic edema is prevalent, identified as hypodense brain tissue. Occasionally, the edema is extensive and, as it predominantly affects white matter, can resembles fingers of low attenuation. Edema, however, is absent in approximately $50 \%$ of cases because of the neoplasm's slow growth. At this patient we can see the calcification as dense. ${ }^{2}$

The World Health Organization (WHO) classifies meningiomas into 15 subtypes under 3 major categories: ${ }^{2,4}$

- Grade I (typical or benign), representing $88-94 \%$ of cases

- Grade II (atypical), i.e. meningiomas with 4-19 mitoses per 10 high power fields (HPF), representing $5-7 \%$ of cases 
- Grade III (anaplastic or malignant), $>20$ cells per $10 \mathrm{HPF}$, representing 1$2 \%$ of cases

The common histologic subtypes of ossified meningioma are transitional, psammomatous, and metaplastic. This densely calcified tumor is characterized by the presence of numerous psammoma bodies. Based on classification above, this tumor is classified as a benign meningioma (WHO grade one). It is usually presented as an extra-axial calcified mass lesion, although in some instances the mass is hyperdense on CT without overt calcification (sparser psammoma bodies). ${ }^{4-5}$

It is better for physicians to do MRI for a more accurate diagnosis. However, it is often not applicable due to resource limitation. MRI more accurately evaluates en plaque and posterior fossa meningiomas, which may be missed on CT scanning. CT scanning, however, clearly depicts bony hyperostosis, which may be difficult to appreciate on MRI. Meningiomas arise from the arachnoid membranes, specifically from meningothelial cells. Most meningiomas grow inward toward the brain as discrete well-defined, dural-based masses and are spherical or lobulated in contour. Flat tumors, termed en plaque, infiltrate the dura and grow as a thin carpet or sheet of tumor along the convexity dura, falx, or tentorium. Dural attachment of meningiomas can be pedunculated or broad-based (sessile). Because the pia and arachnoid form a membranous barrier between brain and tumor, some meningiomas grow into the subarachnoid space, but invasion of the brain is infrequent. In this presented case, we could not see the peduncle, because we only did a head CT scan. ${ }^{1,2,5}$
Surgical treatment could provide a partial or complete resection of a tumor, and the overall prognosis will depend on the entirety of the resection. Recurrence rates after a surgery are related to young age $(<50$ years), multiple lesions, calcification extension, and ossification. ${ }^{4}$ There is only one factor for recurrence in this patient, i.e. classification.

\section{CONCLUSION}

Meningioma has many radiologic features, that usually fit with their histopathological findings. Overt calcification in brain CT scan images psammomatous type of meningioma. Beside history taking and physical findings, adequate and prompt radiologic examination will help physician to make a good diagnosis. Well collaboration from clinician, radiologist, and histopathologist is the key to maintain patient's recovery and safety.

\section{REFERENCES}

1. Meyer SP. Differential diagnosis in neuroimaging brain and meningens. Thieme; 2016.

2. Islam O, Hall GJ, Coombs BD, Runge V, Smirniotoulos JG, Creasy JL, et al. Brain meningioma imaging. Emedicine Medscape: 2016; 1-44

3. Khoshnaw KT and Hosn SS Psammomatous meningioma. Radiopaedia. Available at: https://radiopaedia.org/articles/psammo matous-meningioma

4. World Health Organization. WHO Classification of Meningiomas. USA; 2016

5. Chen MYM, Pope TL, Ott DJ. Basic radiology. 2nd ed. USA: Lange; 2011. 
Vol 5 No 3 October 2020

E-ISSN: 2528-410X 